\title{
A STUDY OF THE QUANTITATIVE RELATIONSHIP BETWEEN ANTIDIURETIC HORMONE (VASOPRESSIN) AND THE RENAL TUBULAR REABSORPTION OF WATER ${ }^{1}$
}

\author{
By WALTER HOLLANDER, JR.,2 T. FRANKLIN WILLIAMS, CHRISTOPHER C. \\ FORDHAM, III, AND LOUIS G. WELT
}

\author{
(From the Department of Medicine, University of North Carolina School of Medicine, \\ Chapel Hill, N.C.)
}

(Submitted for publication December 26, 1956; accepted March 14, 1957)

There is little available information regarding the quantitative relationship between the rate of secretion of antidiuretic hormone $(\mathrm{ADH})$ and the rate of reabsorption of water by the renal tubules. From the work of Shannon (1) and Lauson (2) it has been suggested that in both dogs and adult humans the rate of secretion of $\mathrm{ADH}$ is equivalent to the intravenous administration of vasopressin at rates varying from less than 0.1 to more than 0.7 milliunit per hour per kilogram of body weight. Utilizing the endogenous creatinine $U / P$ ratios as an index of the renal response, these authors both demonstrated a curvilinear relationship between the rate of infusion of vasopressin and the response of the kidney. The lowest rates of infusion of vasopressin studied were sufficient to produce a marked reduction in the rate of urine flow and increase in the endogenous creatinine $U / P$ ratios, and there do not appear to be any data which define the quantitative relationship between $\mathrm{ADH}$ and the renal reabsorption of water in the range in which the concentration of the urine is generally less than that of the plasma. The current studies were designed to establish this relationship with the additional hope that its characteristics might provide some insight with respect to the mode of action of ADH.

${ }^{1}$ Work aided by Research Grant H-1301 (C3), from the National Institutes of Health, United States Public Health Service, and by a contract with the Office of the Surgeon General, United States Army (DA-49-007-MD-369).

2 Work done while a post-doctoral research fellow of the United States Public Health Service.

3 Work done while a post-doctoral research fellow of the Life Insurance Medical Research Fund.

4 Work done while a trainee of the Institute of Arthritis and Metabolism, National Institutes of Health, United States Public Health Service.

\section{PROCEDURE AND METHODS}

Each experiment was designed to compare the rate of urine flow $(V)$ and the clearance of free water $\left(\mathrm{C}_{\mathrm{B}_{2}} \mathrm{O}\right)$ (3) during maximal water diuresis with the urine flow and clearance of free water during the infusion of vasopressin, at one or more constant rates.

The subjects were all apparently healthy young adult men of ages twenty to thirty-five. No food or water was allowed for 10 to 12 hours prior to all but three of the experiments. In three studies (R.W., D.R., and E.D., $1 / 10 / 55$ ), an attempt was made to increase (R.W. and E.D.) or decrease (D.R.) the rate of solute excretion. R.W. and E.D. (1/10/55) received 25 grams of sodium chloride per day added to their diets for two days prior to the study, and a portion thereof on the morning of the study upon arising. D.R. received only water $a d l i b$. plus 200 grams of sucrose per day for two days prior to the study. No smoking was permitted for $\mathbf{8}$ hours prior to or during each experiment. All experiments were started between $7: 30$ and 8:30 A.M. and lasted from 6 to 11 hours, during which the subjects remained recumbent. Urine was collected through an indwelling Foley catheter with the aid of manual supra-pubic pressure. Venous blood was collected under oil through a Cournand needle which remained in an antecubital or forearm vein throughout each study. Approximately $0.25 \mathrm{ml}$. of heparin $(1: 1,000)$ was injected into the Cournand needle after each blood sample was obtained. No food or water was ingested during the experiments.

The basic design of the studies was as follows: In order to suppress the endogenous secretion of $\mathrm{ADH}$ as completely as possible, a positive balance of water of approximately one liter was produced in each subject, usually during the first hour, by the intravenous infusion of a solution of $3 \frac{1}{2}$ per cent dextrose, $3 \frac{1}{2}$ per cent invert sugar, or 5 per cent invert sugar, in water. This positive balance was subsequently maintained by adjusting the rate of infusion so that it approximated the rate of urine flow (infusion controlled by an Omega Machine Company "blood" pump which compresses the infusion tubing). In some experiments a maximal water diuresis was observed initially, followed by one or more constant infusions of vasopressin. In other experiments this order was reversed, and in a few studies an infusion of vasopressin was bracketed by periods of maximal water diuresis. In one subject (R.S.), one rate of infusion of vasopressin (0.5 milliunit 
per hour) was compared with a preceding maximal water diuresis; another rate of infusion (1.0 milliunit per hour) was compared with a subsequent maximal water diuresis. All infusions of vasopressin and all maximal water diureses were continued until an equilibrium appeared to have been achieved; i.e., an approximately constant rate of urine flow for at least 2 to 3 collection periods of 10 minutes or more. Venous blood was obtained through the Cournand needle near the end of each such series of "equilibrium" periods, usually at the start of the last period. Studies were eliminated if emotional factors appeared to have influenced the results, but it is obviously impossible to know that they were not involved in those reported.

Two separate lots of Pitressin ${ }^{85}$ were used: the first (RH-303 M, studies 1 through 5) was several years old but was used only during a three-month period and was reassayed (pressor activity) by the Parke, Davis and Company laboratories during that period; the second lot ( $\mathbf{P}$ 189750, studies 6 through 9) was generously supplied new by Parke, Davis and Company and was assayed by them before and after the experiments. The first lot apparently had deteriorated by about $\mathbf{5 0}$ per cent, and this fact has been incorporated in the rates of infusion reported. The pre- and post-experimental assays on the second lot indicated no significant decrease in pressor potency. The appropriate dilutions of vasopressin were all prepared from freshly opened ampoules in $\mathbf{5}$ per cent invert sugar and water immediately before infusion, and were all administered intravenously at a constant rate of approximately $1 \mathrm{ml}$. per minute controlled by a Bowman finger-type infusion pump. The constancy of this pump was studied on several occasions and showed a variation of less than $0.1 \mathrm{ml}$. per minute over a period of 8 or more hours. The rates of infusion were checked in each experiment by timing the infusions and by weighing the bottles of vasopressin before and after completion of the infusions.

Inulin was administered intravenously as a $2 \frac{1}{2}$ per cent solution in $\mathbf{5}$ per cent invert sugar and water at a constant rate of approximately $1 \mathrm{ml}$. per minute. Because of technical difficulties subsequent to the experiments, most of the analyses for inulin were unsatisfactory and clearances of inulin cannot be reported.

The osmolal concentrations of the serum and urine were measured on the day of the study with a Fiske Freezing Point Osmometer which was calibrated to read osmolality at the time of each set of determinations (4). A qualitative test for glucose in the urine was performed by the standard Benedict's method.

The osmolal clearance $\left(\mathrm{C}_{\mathrm{osm}}\right)$ (3) and the clearance of free water $\left(\mathrm{C}_{\mathrm{H}_{2} \mathrm{O}}\right)$ (3) were calculated from the following formulas:

$$
\mathrm{C}_{\mathrm{osm}}=\frac{[\mathrm{U}]_{\mathrm{osm}} \mathrm{V}}{[\mathrm{P}]_{\mathrm{osm}}} \quad \mathrm{C}_{\mathrm{H}_{2} \mathrm{O}}=\mathrm{V}-\mathrm{C}_{\mathrm{oom}}
$$

where $[U]_{\text {oem }}=$ osmolal concentration of urine, $[P]_{\text {oem }}=$

s Both lots were derived from an indeterminate mixture of hog and beef pituitaries and were supplied through the courtesy of Dr. A. C. Bratton, Jr., of Parke, Davis and Company. osmolal concentration of serum, and $\mathrm{V}=$ urine flow in $\mathrm{ml}$. per minute.

The change in free water clearance $\left(\Delta \mathrm{C}_{\mathrm{H}_{2} \mathrm{O}}\right)$ associated with each rate of infusion of vasopressin was calculated by subtracting the average free water clearance observed at "equilibrium" during the infusion of vasopressin from the free water clearance obtained in the same experiment during maximal water diuresis $\left(\mathrm{C}_{\mathrm{H}_{2} \mathrm{O}}{ }^{\max }-\mathrm{C}_{\mathrm{H}_{2} \mathrm{O}}=\Delta \mathrm{C}_{\mathrm{H}_{2} \mathrm{O}}\right)$. The values for $\Delta \mathrm{C}_{\mathrm{H}_{2} \mathrm{O}}$ have all been corrected to a standard surface area of 1.73 square meters $\left(\Delta C^{\prime}{ }_{H_{2} \mathrm{O}}\right)$, the surface area of subjects being estimated from their height and weight. The change in flow of urine ( $\Delta$ flow) and the change in $\mathrm{C}_{\text {osm }}\left(\Delta \mathrm{C}_{\text {orm }}\right)$ have been calculated in the same manner as has $\Delta \mathbf{C}_{\mathrm{H}_{2} \mathrm{O}}$.

The method of analysis of variance was utilized to examine the adequacy of the calculated regression equations.

\section{RESULTS}

The rates of excretion of urine, the osmolal concentrations of the urine and serum, and the rate of excretion of solutes for all "equilibrium" periods are shown in Table $I$. The average values during "equilibrium" of $\mathrm{V},[\mathrm{U}]_{\mathrm{osm}}, \mathrm{C}_{\mathrm{osm}}, \mathrm{C}_{\mathrm{H}_{2} \mathrm{O}}$, $\Delta$ flow, $\Delta \mathrm{C}_{\text {osm }}, \Delta \mathrm{C}_{\mathrm{H}_{3} \mathrm{O}}$ and $\Delta \mathrm{C}_{\mathrm{H}_{3} \mathrm{O}}^{\prime}$ are presented in Table II. The urine flow and free water clearance during maximum water diuresis varied significantly among subjects. Similarly, if all subjects are considered, the urine flow and free water clearance during an infusion of vasopressin bore no absolute relationship to the rate of the infusion. Except for three subjects (R.W. 10/ $17 / 54$, E.D. $1 / 10 / 55$, and D.R. $2 / 23 / 55$ ), all values of $\mathrm{C}_{\mathrm{osm}}$ during maximal water diuresis $\left(\mathrm{C}^{\circ}{ }_{\mathrm{osm}}\right)$ were between 2.3 and $4.0 \mathrm{ml}$. per minute. The exceptions $\left(\mathrm{C}^{\circ}{ }_{\text {osm }}=7.0,7.2,1.9\right.$, and 1.7 ml. per minute) were produced intentionally as explained under "methods" in an effort to examine the effect of the rate of solute excretion on the quantitative behaviour of vasopressin. In twelve of the seventeen observations, $\Delta \mathrm{C}_{\text {osm }}$ was no greater than $0.5 \mathrm{ml}$. per minute. There was no demonstrated glycosuria during the "equilibrium" periods of most studies; in a few, during the relatively higher rates of infusion of vasopressin, slight glycosuria was detected but never exceeded approximately 1 gram per cent. It is therefore unlikely that glucose was ever a major constituent of the urinary solutes.

The data in Table II show a considerable degree of biologic variation, despite which it can be seen that there was a graded curvilinear relationship between the rate of infusion of vasopressin and the renal response. The relationship 
TABLE I

The effects of various rates of infusion of vasopressin-Measured and derived data during all "equilibrium" collection periods

\begin{tabular}{|c|c|c|c|c|c|c|}
\hline \multirow{2}{*}{$\begin{array}{c}\text { Subject } \\
\text { Date } \\
\text { Weight } \\
\text { Height } \\
\text { Est. 8.a. }\end{array}$} & \multirow[b]{2}{*}{$\begin{array}{l}\text { Rate of } \\
\text { infusion of } \\
\text { vasopressin }\end{array}$} & \multirow[b]{2}{*}{ Time } & \multicolumn{3}{|c|}{ Urine } & \multirow{2}{*}{$\frac{\text { Serum }}{\begin{array}{c}\text { Solute } \\
\text { concentration }\end{array}}$} \\
\hline & & & Flow & $\begin{array}{c}\text { Solute } \\
\text { concentration }\end{array}$ & $\begin{array}{l}\text { Rate of } \\
\text { solute } \\
\text { excretion }\end{array}$ & \\
\hline \multirow{5}{*}{$\begin{array}{l}\text { R. S. } \\
8 / 22 / 54 \\
75.80 \mathrm{Kg} . \\
180 \mathrm{~cm} . \\
1.93 \mathrm{sq} . \mathrm{M} .\end{array}$} & $m U . /$ hour & mineutes & $m b . / m i x$ & milliosmolal & $\begin{array}{l}\text { micro-osmoles/ } \\
\text { mix. }\end{array}$ & milliosmolal \\
\hline & $\begin{array}{l}\mathbf{0} \\
\mathbf{0} \\
\mathbf{0}\end{array}$ & $\begin{array}{l}156-168 \\
169-178 \\
179-191\end{array}$ & $\begin{array}{l}14.2 \\
14.6 \\
14.6\end{array}$ & $\begin{array}{l}48.0 \\
47.0 \\
45.0\end{array}$ & $\begin{array}{l}682 \\
686 \\
657\end{array}$ & 272 \\
\hline & $\begin{array}{l}0.5 \\
0.5 \\
0.5\end{array}$ & $\begin{array}{l}248-263 \\
264-278 \\
279-292\end{array}$ & $\begin{array}{l}11.5 \\
11.7 \\
11.6\end{array}$ & $\begin{array}{l}55.0 \\
57.0 \\
63.0\end{array}$ & $\begin{array}{l}632 \\
667 \\
731\end{array}$ & 271 \\
\hline & $\begin{array}{l}1.0 \\
1.0 \\
1.0\end{array}$ & $\begin{array}{l}355-373 \\
374-391 \\
392-416\end{array}$ & $\begin{array}{l}13.6 \\
13.7 \\
13.5\end{array}$ & $\begin{array}{l}60.0 \\
60.0 \\
57.0\end{array}$ & $\begin{array}{l}816 \\
822 \\
770\end{array}$ & 269 \\
\hline & $\begin{array}{l}0 \\
0 \\
0\end{array}$ & $\begin{array}{l}464-484 \\
485-507 \\
508-518\end{array}$ & $\begin{array}{l}16.4 \\
17.6 \\
17.0\end{array}$ & $\begin{array}{l}46.0 \\
44.0 \\
45.0\end{array}$ & $\begin{array}{l}754 \\
774 \\
765\end{array}$ & 265 \\
\hline \multirow{4}{*}{$\begin{array}{l}\text { D. D. } \\
8 / 26 / 54 \\
70.05 \mathrm{Kg} . \\
190 \mathrm{~cm} . \\
1.96 \mathrm{sq} . \mathrm{M} .\end{array}$} & $\begin{array}{l}9.8 \\
9.8 \\
9.8\end{array}$ & $\begin{array}{l}155-169 \\
170-183 \\
184-199\end{array}$ & $\begin{array}{l}7.0 \\
7.1 \\
7.0\end{array}$ & $\begin{array}{r}100.0 \\
97.0 \\
99.0\end{array}$ & $\begin{array}{l}700 \\
689 \\
693\end{array}$ & 275 \\
\hline & $\begin{array}{l}2.5 \\
2.5 \\
2.5\end{array}$ & $\begin{array}{l}241-260 \\
261-274 \\
275-289\end{array}$ & $\begin{array}{l}13.1 \\
13.8 \\
14.2\end{array}$ & $\begin{array}{l}56.0 \\
54.9 \\
51.9\end{array}$ & $\begin{array}{l}734 \\
758 \\
738\end{array}$ & 273.5 \\
\hline & $\begin{array}{l}1.4 \\
1.4\end{array}$ & $\begin{array}{l}409-422 \\
423-437\end{array}$ & $\begin{array}{l}15.0 \\
16.6\end{array}$ & $\begin{array}{l}43.0 \\
48.0\end{array}$ & $\begin{array}{l}645 \\
798\end{array}$ & $270.5 \ddagger$ \\
\hline & $\begin{array}{l}\mathbf{0} \\
\mathbf{0}\end{array}$ & $\begin{array}{l}485-499 \\
500-508\end{array}$ & $\begin{array}{l}19.3 \\
19.4\end{array}$ & $\begin{array}{l}39.0 \\
37.0\end{array}$ & $\begin{array}{l}753 \\
718\end{array}$ & 269 \\
\hline \multirow{3}{*}{$\begin{array}{l}\text { J. A. } \\
9 / 20 / 54 \\
92.20 \mathrm{Kg} . \\
187 \mathrm{~cm} . \\
2.18 \mathrm{sq} . \mathrm{M} .\end{array}$} & $\begin{array}{l}9.3 \\
9.3 \\
9.3\end{array}$ & $\begin{array}{l}186-206 \\
207-225 \\
226-247\end{array}$ & $\begin{array}{l}1.7 \\
1.7 \\
1.9\end{array}$ & $\begin{array}{l}318.0 \\
293.0 \\
313.0\end{array}$ & $\begin{array}{l}540 \\
498 \\
594\end{array}$ & 269 \\
\hline & $\begin{array}{l}4.7 \\
4.7 \\
4.7\end{array}$ & $\begin{array}{l}384-404 \\
405-426 \\
427-449\end{array}$ & $\begin{array}{l}2.9 \\
2.5 \\
3.8\end{array}$ & $\begin{array}{l}144.0 \\
205.0 \\
141.0\end{array}$ & $\begin{array}{l}417 \\
513 \\
536\end{array}$ & 269 \\
\hline & $\begin{array}{l}\mathbf{0} \\
\mathbf{0}\end{array}$ & $\begin{array}{l}553-565 \\
566-575\end{array}$ & $\begin{array}{l}15.1 \\
14.0\end{array}$ & $\begin{array}{l}43.1 \\
43.0\end{array}$ & $\begin{array}{l}652 \\
602\end{array}$ & 270 \\
\hline \multirow{3}{*}{$\begin{array}{l}\text { E. D. } \\
9 / 30 / 54 \\
74.55 \mathrm{Kg} . \\
177 \mathrm{~cm} . \\
1.90 \mathrm{sq} . \mathrm{M} .\end{array}$} & $\begin{array}{l}7.2 \\
7.2\end{array}$ & $\begin{array}{l}113-137 \\
138-158\end{array}$ & $\begin{array}{l}6.6 \\
6.3\end{array}$ & $\begin{array}{l}190.0 \\
177.0\end{array}$ & $\begin{array}{l}1,254 \\
1,115\end{array}$ & 271 \\
\hline & $\begin{array}{l}2.1 \\
2.1 \\
2.1\end{array}$ & $\begin{array}{l}280-300 \\
301-317 \\
318-333\end{array}$ & $\begin{array}{l}11.8 \\
11.8 \\
12.1\end{array}$ & $\begin{array}{l}60.8 \\
60.8 \\
66.1\end{array}$ & $\begin{array}{l}718 \\
718 \\
800\end{array}$ & 270 \\
\hline & $\begin{array}{l}0 \\
0 \\
0 \\
0\end{array}$ & $\begin{array}{l}478-495 \\
496-511 \\
512-526 \\
527-538\end{array}$ & $\begin{array}{l}22.2 \\
24.5 \\
20.5 \\
19.6\end{array}$ & $\begin{array}{l}52.0 \\
48.0 \\
44.0 \\
46.0\end{array}$ & $\begin{array}{r}1,155 \\
1,176 \\
902 \\
900\end{array}$ & 266 \\
\hline \multirow[t]{2}{*}{$\begin{array}{l}\text { R. W. } \\
10 / 17 / 54 \\
74.70 \mathrm{Kg} . \\
181 \mathrm{~cm} . \\
1.94 \text { sq. M. }\end{array}$} & $\begin{array}{l}0 \\
0 \\
0 \\
0\end{array}$ & $\begin{array}{c}57-98 \\
99-110 \\
111-125 \\
126-136\end{array}$ & $\begin{array}{l}24.7 \\
23.7 \\
22.9 \\
23.7\end{array}$ & $\begin{array}{l}88.7 \\
80.1 \\
72.9 \\
62.8\end{array}$ & $\begin{array}{l}2,192 \\
1,900 \\
1,670 \\
1,490\end{array}$ & 276 \\
\hline & $\begin{array}{l}0.4 \\
0.4 \\
0.4\end{array}$ & $\begin{array}{l}216-233 \\
234-253 \\
254-273\end{array}$ & $\begin{array}{l}18.6 \\
18.3 \\
18.7\end{array}$ & $\begin{array}{l}48.8 \\
47.9 \\
48.0\end{array}$ & $\begin{array}{l}909 \\
878 \\
899\end{array}$ & 270 \\
\hline
\end{tabular}


TABLE I-Continued

\begin{tabular}{|c|c|c|c|c|c|c|}
\hline \multirow{2}{*}{$\begin{array}{c}\text { Subject } \\
\text { Date } \\
\text { Weiteht } \\
\text { Helight } \\
\text { Eest. s.a. }\end{array}$} & \multirow[b]{2}{*}{$\begin{array}{l}\text { Rate of } \\
\text { infualon of } \\
\text { vasopressin }\end{array}$} & \multirow[b]{2}{*}{ Time } & \multicolumn{3}{|c|}{ Urine } & \multirow{2}{*}{$\frac{\text { Serum }}{\begin{array}{c}\text { Solute } \\
\text { concentration }\end{array}}$} \\
\hline & & & Flow & $\begin{array}{c}\text { Solute } \\
\text { concentration }\end{array}$ & $\begin{array}{c}\begin{array}{c}\text { Rate of } \\
\text { oolute } \\
\text { excretion }\end{array}\end{array}$ & \\
\hline \multirow{5}{*}{$\begin{array}{l}\text { W. M. } \\
1 / 3 / 55 \\
76.65 \mathrm{Kg} . \\
190 \mathrm{~cm} . \\
2.06 \text { sq. M. }\end{array}$} & $m U . /$ hour & minutes & $m b . / m i n$. & milliosmalal & $\begin{array}{l}\text { micro-osmoles/ } \\
\text { mix. }\end{array}$ & milliosmolal \\
\hline & $\begin{array}{l}1.9 \\
1.9 \\
1.9\end{array}$ & $\begin{array}{l}383-402 \\
403-422 \\
423-439\end{array}$ & $\begin{array}{r}9.9 \\
8.5 \\
10.9\end{array}$ & $\begin{array}{r}85.5 \\
102.0 \\
87.8\end{array}$ & $\begin{array}{l}847 \\
867 \\
958\end{array}$ & 266 \\
\hline & $\begin{array}{l}1.8 \\
1.8\end{array}$ & $\begin{array}{l}161-187 \\
188-223\end{array}$ & $\begin{array}{l}1.85 \\
1.1\end{array}$ & $\begin{array}{l}397.0 \\
436.0\end{array}$ & $\begin{array}{l}734 \\
480\end{array}$ & $276 \ddagger$ \\
\hline & $\begin{array}{l}\mathbf{0} \\
\mathbf{0}\end{array}$ & $\begin{array}{l}373-395 \\
396-415\end{array}$ & $\begin{array}{l}12.1 \\
10.9\end{array}$ & $\begin{array}{l}78.9 \\
70.0\end{array}$ & $\begin{array}{l}955 \\
763\end{array}$ & 268 \\
\hline & $\begin{array}{l}1.8 \\
1.8 \\
1.8\end{array}$ & $\begin{array}{l}493-507 \\
508-523 \\
524-540\end{array}$ & $\begin{array}{l}5.3 \\
4.9 \\
4.9\end{array}$ & $\begin{array}{l}167.0 \\
175.0 \\
171.0\end{array}$ & $\begin{array}{l}884 \\
856 \\
838\end{array}$ & 265 \\
\hline \multirow{2}{*}{$\begin{array}{l}\text { E. D.* } \\
1 / 10 / 55 \\
75.90 \mathrm{Kg} . \\
177 \mathrm{~cm} . \\
1.92 \mathrm{sq} . \mathrm{M} .\end{array}$} & $\begin{array}{l}15.5 \\
15.5 \\
15.5\end{array}$ & $\begin{array}{l}130-152 \\
153-172 \\
173-192\end{array}$ & $\begin{array}{l}4.4 \\
4.2 \\
4.0\end{array}$ & $\begin{array}{l}501.0 \\
542.0 \\
565.0\end{array}$ & $\begin{array}{l}2,205 \\
2,278 \\
2,258\end{array}$ & 274 \\
\hline & $\begin{array}{l}\mathbf{0} \\
\mathbf{0} \\
\mathbf{0}\end{array}$ & $\begin{array}{l}360-376 \\
377-399 \\
400-417 \\
418-427\end{array}$ & $\begin{array}{l}28.8 \\
28.7 \\
28.8 \\
29.8\end{array}$ & $\begin{array}{l}72.9 \\
67.9 \\
64.0 \\
59.9\end{array}$ & $\begin{array}{l}2,102 \\
1,951 \\
1,844 \\
1,786\end{array}$ & 268 \\
\hline \multirow{3}{*}{$\begin{array}{l}\text { D. R. } \dagger \\
2 / 23 / 55 \\
73.97 \mathrm{Kg} . \\
182 \mathrm{~cm} . \\
1.94 \mathrm{sq} . \mathrm{M} .\end{array}$} & $\begin{array}{l}\mathbf{0} \\
\mathbf{0} \\
\mathbf{0}\end{array}$ & $\begin{array}{l}149-168 \\
169-189 \\
190-207\end{array}$ & $\begin{array}{r}9.5 \\
11.2 \\
10.0\end{array}$ & $\begin{array}{l}53.0 \\
51.9 \\
52.7\end{array}$ & $\begin{array}{l}504 \\
582 \\
527\end{array}$ & 278 \\
\hline & $\begin{array}{l}1.7 \\
1.7 \\
1.7\end{array}$ & $\begin{array}{l}328-349 \\
350-369 \\
370-389\end{array}$ & $\begin{array}{l}3.4 \\
3.3 \\
3.7\end{array}$ & $\begin{array}{l}112.0 \\
117.0 \\
109.0\end{array}$ & $\begin{array}{l}382 \\
385 \\
402\end{array}$ & 270 \\
\hline & $\begin{array}{l}\mathbf{0} \\
\mathbf{0} \\
\mathbf{0} \\
\mathbf{0}\end{array}$ & $\begin{array}{l}461-479 \\
480-499 \\
500-519 \\
520-530\end{array}$ & $\begin{array}{l}9.1 \\
8.3 \\
8.5 \\
9.6\end{array}$ & $\begin{array}{l}55.1 \\
53.7 \\
52.0 \\
50.7\end{array}$ & $\begin{array}{l}502 \\
446 \\
442 \\
487\end{array}$ & 271 \\
\hline \multirow{2}{*}{$\begin{array}{l}\text { T. C. } \\
5 / 6 / 55 \\
82.65 \mathrm{Kg} . \\
188 \mathrm{~cm} . \\
2.1 \text { sq. M. }\end{array}$} & $\begin{array}{l}10.7 \\
10.7 \\
10.7\end{array}$ & $\begin{array}{l}305-324 \\
325-344 \\
345-364\end{array}$ & $\begin{array}{l}1.2 \\
0.9 \\
0.9\end{array}$ & $\begin{array}{l}475.0 \\
584.0 \\
600.0\end{array}$ & $\begin{array}{l}570 \\
526 \\
540\end{array}$ & 271 \\
\hline & $\begin{array}{l}\mathbf{0} \\
\mathbf{0} \\
\mathbf{0}\end{array}$ & $\begin{array}{l}497-511 \\
512-526 \\
527-540\end{array}$ & $\begin{array}{l}13.0 \\
12.9 \\
13.2\end{array}$ & $\begin{array}{l}55.0 \\
51.8 \\
51.0\end{array}$ & $\begin{array}{l}715 \\
669 \\
674\end{array}$ & 275 \\
\hline
\end{tabular}

* High sodium chloride intake prior to study (see text).

t Low solute intake prior to study (see text).

$\mp$ An interpolated value.

may be expressed by at least three simple regression equations, the renal response being expressed as $\Delta \mathrm{C}_{\mathrm{H}_{2} \mathrm{O}}^{\prime}$ since it appears reasonable to assume that the change in free water clearance represents the increase in the reabsorption of water by the renal tubules which results from each particular infusion of vasopressin (see Discussion). If $\Delta \mathrm{C}_{\text {oom }}$ were zero, $\Delta \mathrm{C}_{\mathrm{H}_{2} \mathrm{O}}$ would of course equal $\Delta$ flow. The equations $(1,2$, and 3$)$ and the results of analysis of variance are shown in Table III-A. All three equations are highly significant in a statistical sense, but at such extremely high values of " $F$," statistical considerations alone cannot determine which expression most accurately defines the relationship. Furthermore, the derivation of these particular equations is in no sense intended to imply that they represent the only expressions which will describe the data, since it is certain that many other more complex equations involving two or more constants would do so as well.

In order to minimize any effect which a change 
TABLE II

The effects of various rates of infusion of vasopressin-Average "equilibrium" values

\begin{tabular}{|c|c|c|c|c|c|c|c|c|c|}
\hline \multirow[b]{2}{*}{$\begin{array}{c}\text { Subject } \\
\text { Date }\end{array}$} & \multirow{2}{*}{$\begin{array}{c}\text { Rate of } \\
\text { infusion of } \\
\text { vasopreasin }\end{array}$} & \multicolumn{8}{|c|}{ Urine } \\
\hline & & Flow & $\begin{array}{c}\text { Solute } \\
\text { concentration }\end{array}$ & $C_{0}$ & $\mathrm{CH}_{\mathrm{B} O \mathrm{O}}$ & $\Delta$ Flow & $\Delta C_{0}$ om & $\Delta \mathrm{C}_{\mathrm{B} 9 \mathrm{O}}$ & $\Delta C^{\prime}{ }_{8} \mathrm{O}$ \\
\hline \multirow[t]{2}{*}{${ }_{8 / 22 / 54}^{\text {R.S. }}$} & $\begin{array}{c}m U . / h r . \\
0 \\
0.5\end{array}$ & $\begin{array}{c}\text { ml./min. } \\
14.5 \\
11.6\end{array}$ & $\begin{array}{c}\text { milliosmolal } \\
47 \\
58\end{array}$ & $\begin{array}{c}\text { ml. } / \text { min. } \\
2.5 \\
2.5\end{array}$ & $\begin{array}{c}\text { ml. } / \text { min. } \\
12.0 \\
9.1\end{array}$ & $\begin{array}{c}\text { ml. } / \text { min. } \\
2.9\end{array}$ & $\begin{array}{c}m l . / m i n . \\
0\end{array}$ & $\begin{array}{c}m l . / m i n . \\
2.9\end{array}$ & $\begin{array}{c}m l . / m i n . \\
2.6\end{array}$ \\
\hline & $\begin{array}{c}0 \\
1.0\end{array}$ & $\begin{array}{l}17.0 \\
13.6\end{array}$ & $\begin{array}{l}45 \\
59\end{array}$ & $\begin{array}{l}2.9 \\
3.0\end{array}$ & $\begin{array}{l}14.1 \\
10.6\end{array}$ & 3.4 & -0.1 & 3.5 & 3.1 \\
\hline $\begin{array}{l}\text { D. D. } \\
8 / 26 / 54\end{array}$ & $\begin{array}{c}0 \\
1.4 \\
2.5 \\
9.8\end{array}$ & $\begin{array}{r}19.4 \\
15.8 \\
13.7 \\
7.0\end{array}$ & $\begin{array}{l}38 \\
45 \\
54 \\
99\end{array}$ & $\begin{array}{l}2.8 \\
2.7 \\
2.7 \\
2.5\end{array}$ & $\begin{array}{r}16.6 \\
13.1 \\
11.0 \\
4.5\end{array}$ & $\begin{array}{r}3.6 \\
5.7 \\
12.4\end{array}$ & $\begin{array}{l}0.1 \\
0.1 \\
0.3\end{array}$ & $\begin{array}{r}3.5 \\
5.6 \\
12.1\end{array}$ & $\begin{array}{r}3.1 \\
4.9 \\
10.7\end{array}$ \\
\hline $\begin{array}{l}\text { J. A. A } \\
9 / 20 / 54\end{array}$ & $\begin{array}{c}0 \\
4.7 \\
9.3\end{array}$ & $\begin{array}{r}14.7 \\
3.1 \\
1.8\end{array}$ & $\begin{array}{r}43 \\
163 \\
309\end{array}$ & $\begin{array}{l}2.3 \\
1.8 \\
2.0\end{array}$ & $\begin{array}{r}12.4 \\
1.3 \\
-0.2\end{array}$ & $\begin{array}{l}11.6 \\
12.9\end{array}$ & $\begin{array}{l}0.5 \\
0.3\end{array}$ & $\begin{array}{l}11.1 \\
12.6\end{array}$ & $\begin{array}{r}8.8 \\
10.0\end{array}$ \\
\hline E. D/30/54 & $\begin{array}{c}0 \\
2.1 \\
7.2\end{array}$ & $\begin{array}{r}21.9 \\
11.9 \\
6.5\end{array}$ & $\begin{array}{r}48 \\
62 \\
184\end{array}$ & $\begin{array}{l}3.9 \\
2.8 \\
4.4\end{array}$ & $\begin{array}{r}18.0 \\
9.1 \\
2.1\end{array}$ & $\begin{array}{l}10.0 \\
15.4\end{array}$ & $\begin{array}{r}1.1 \\
-0.5\end{array}$ & $\begin{array}{r}8.9 \\
15.9\end{array}$ & $\begin{array}{r}8.1 \\
14.5\end{array}$ \\
\hline $\begin{array}{l}\text { R. W. W } \\
10 / 17 / 54\end{array}$ & $\begin{array}{c}0 \\
0.4 \\
1.9\end{array}$ & $\begin{array}{r}24.1 \\
18.5 \\
9.7\end{array}$ & $\begin{array}{l}81 \\
48 \\
92\end{array}$ & $\begin{array}{l}7.0 \\
3.3 \\
3.3\end{array}$ & $\begin{array}{r}17.1 \\
15.2 \\
6.4\end{array}$ & $\begin{array}{r}5.6 \\
14.4\end{array}$ & $\begin{array}{l}3.7 \\
3.7\end{array}$ & $\begin{array}{r}1.9 \\
10.7\end{array}$ & $\begin{array}{l}1.7 \\
9.5\end{array}$ \\
\hline \multirow[t]{2}{*}{ W. M. $_{1 / 3 / 55}$} & $\begin{array}{c}0 \\
1.8\end{array}$ & $\begin{array}{r}11.5 \\
1.4\end{array}$ & $\begin{array}{r}75 \\
419\end{array}$ & $\begin{array}{l}3.2 \\
2.1\end{array}$ & $\begin{array}{r}8.3 \\
-0.7\end{array}$ & 10.1 & 1.1 & 9.0 & 7.6 \\
\hline & $\begin{array}{c}0 \\
1.8\end{array}$ & $\begin{array}{r}11.5 \\
5.0\end{array}$ & $\begin{array}{r}75 \\
171\end{array}$ & $\begin{array}{l}3.2 \\
3.2\end{array}$ & $\begin{array}{l}8.3 \\
1.8\end{array}$ & 6.5 & 0 & 6.5 & 5.5 \\
\hline E. D. ${ }_{1 / 10 / 55}^{*}$ & $\begin{array}{c}0 \\
15.5\end{array}$ & $\begin{array}{r}28.9 \\
4.2\end{array}$ & $\begin{array}{r}67 \\
534\end{array}$ & $\begin{array}{l}7.2 \\
8.2\end{array}$ & $\begin{array}{r}21.7 \\
-4.0\end{array}$ & 24.7 & -1.0 & 25.7 & 23.4 \\
\hline \multirow[t]{2}{*}{$\begin{array}{l}\text { D. R. } \dagger \\
2 / 23 / 55\end{array}$} & $\begin{array}{c}0 \\
1.7\end{array}$ & $\begin{array}{r}10.2 \\
3.4\end{array}$ & $\begin{array}{r}53 \\
113\end{array}$ & $\begin{array}{l}1.9 \\
1.4\end{array}$ & $\begin{array}{l}8.3 \\
2.0\end{array}$ & 6.8 & 0.5 & 6.3 & 5.6 \\
\hline & $\begin{array}{c}0 \\
1.7\end{array}$ & $\begin{array}{l}8.7 \\
3.4\end{array}$ & $\begin{array}{r}53 \\
113\end{array}$ & $\begin{array}{l}1.7 \\
1.4\end{array}$ & $\begin{array}{l}7.0 \\
2.0\end{array}$ & 5.3 & 0.3 & 5.0 & 4.5 \\
\hline T. C. $_{5 / 6 / 55}$ & $\begin{array}{c}0 \\
10.7\end{array}$ & $\begin{array}{r}13.0 \\
1.0\end{array}$ & $\begin{array}{r}53 \\
553\end{array}$ & $\begin{array}{l}2.5 \\
2.0\end{array}$ & $\begin{array}{r}10.5 \\
-1.0\end{array}$ & 12.0 & 0.5 & 11.5 & 9.5 \\
\hline
\end{tabular}

* High $\mathrm{NaCl}$ diet (see text).

† Low solute diet (see text).

TABLE III

Regression equations and the results of analysis of variance

\begin{tabular}{|c|c|c|c|}
\hline \multirow{2}{*}{\multicolumn{2}{|c|}{ Equation }} & \multicolumn{2}{|c|}{ Analysis of variance } \\
\hline & & "F"* & $\begin{array}{l}\text { Variation in data } \\
\text { "removed" by } \\
\text { the equation } \\
\text { (\% of total) }\end{array}$ \\
\hline \multirow{2}{*}{ (A) } & All data & & \\
\hline & $\begin{array}{l}\text { (1) } \Delta C_{H_{20}}^{\prime}=3.89+9.39 \log \text { (vasopressin rate) } \\
\text { (2) } \log \Delta C^{\prime}{ }_{H_{20}}=0.570+0.564 \log \text { (vasopressin rate) } \\
\text { (3) } 1 / \Delta C^{\prime}{ }_{H_{50}}=0.071+0.196 \text { (1/vasopressin rate) }\end{array}$ & $\begin{array}{r}32.0 \\
63.6 \\
108\end{array}$ & $\begin{array}{l}68.1 \\
80.9 \\
87.8\end{array}$ \\
\hline \multirow[t]{2}{*}{ (B) } & Data associated with $\Delta \mathrm{C}_{\text {oem }}$ of $0.5 \mathrm{ml}$. or less & & \\
\hline & $\begin{array}{l}\text { (4) } \Delta C^{\prime}{ }_{H_{20}}^{\prime}=3.44+7.58 \log \text { (vasopressin rate) } \\
\text { (5) } \log \Delta C^{\prime}{ }_{H_{20}}=0.542+0.519 \log \text { (vasopressin rate) } \\
\text { (6) } 1 / \Delta C_{H_{2} O}^{\prime}=0.098+0.173 \text { (1/vasopressin rate) }\end{array}$ & $\begin{array}{l}37.9 \\
70.6 \\
42.1\end{array}$ & $\begin{array}{l}79.1 \\
87.6 \\
80.7\end{array}$ \\
\hline
\end{tabular}

*The probability of "F" occurring by chance is less than 0.001 for each of the equations. 


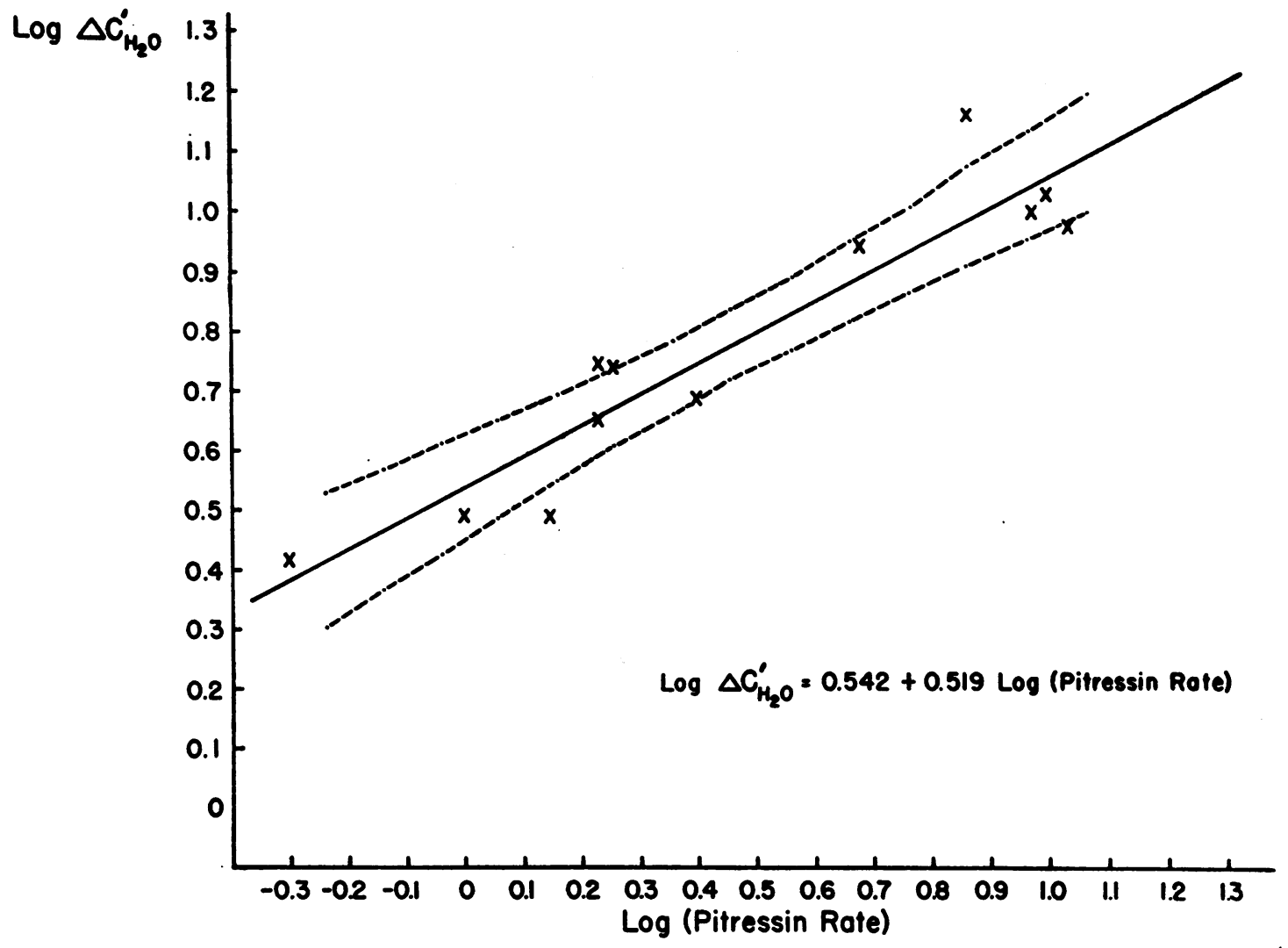

Fig. 1. The Relationship Between $\Delta C^{\prime}{ }_{2} \mathrm{O}$ (ML. Per Minute) and the Rate of Infusion of

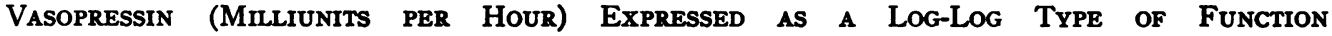

The dotted lines represent the 95 per cent confidence limits for the mean values of $\log \Delta \mathrm{C}_{\mathrm{H}_{2} \mathrm{O}}^{\prime}$ (not for individual values).

in the rate of excretion of solutes might have on $\Delta \mathrm{C}_{\mathrm{H}_{2} \mathrm{O}}^{\prime}$, the data have been reconsidered after eliminating the five studies associated with a $\Delta \mathrm{C}_{\text {osm }}$ of more than $0.5 \mathrm{ml}$. per minute. This also eliminates four of the five studies in which $\mathrm{C}^{\circ}{ }_{\text {omm }}$ was greater than $3.5 \mathrm{ml}$. per minute. The resulting regression equations $(4,5$, and 6$)$ are shown in Table III-B. Equations 5 and 6 are also illustrated in Figures 1 and 2. Again, statistical analysis revealed that the three types of equation are very well approximated by the data. It will also be noted that, if the one point representing a rate of infusion of vasopressin of 0.5 milliunit per hour were omitted, the data would describe a regression equation of the type shown in Figure 2 (Equation 6) with considerably less variation about the line.

That these three types of mathematical expression should all provide such excellent de- scriptions of the data is not surprising because, as shown in Figure 3, these three equations approximate each other quite closely throughout most of the dose range involved in their derivation (0.5 to 10.7 milliunits per hour). Obviously, it would be extremely difficult to differentiate between them in this range on the basis of data as variable as that provided by most biological studies.

It should be emphasized that in all but one study (E.D. 1/10/55) the rates of infusion of vasopressin were less than that required to produce a significantly hypertonic urine and the relationships defined may not be applicable at higher rates of administration of vasopressin.

The method of analysis of variance was utilized to examine the influence of the rate of excretion of solutes on the renal response to vasopressin. The variable considered was the value of $\mathrm{C}_{\text {osm }}$ 


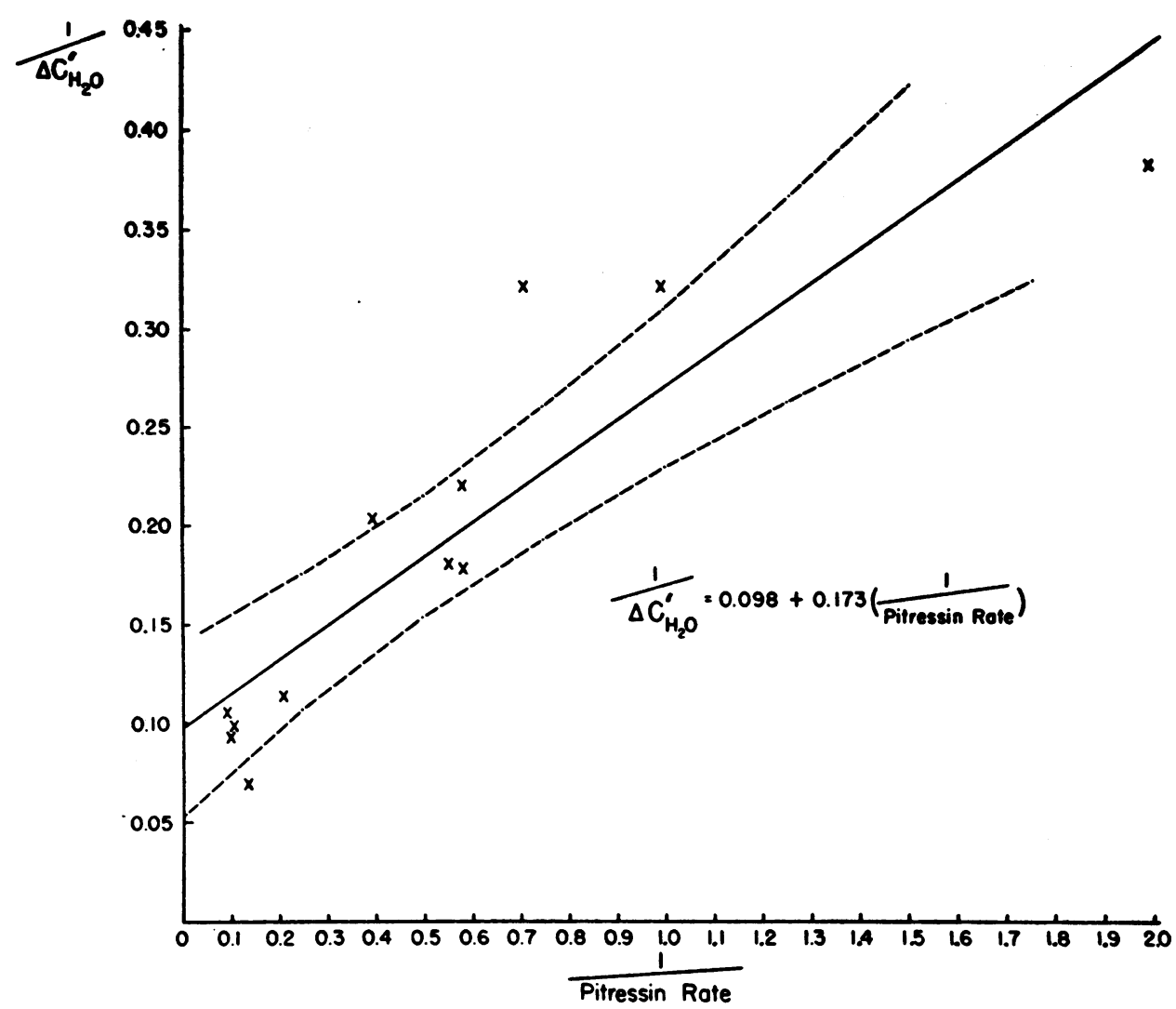

Fig. 2. The Relationship Between $\Delta C^{\prime}{ }_{3} \mathrm{O}$ (ML. PER Minute) and the Rate of INfusion of VASOPRessin (MILliunits Per Hour) EXPressed as a Reciprocal Type of Function The dotted lines represent the 95 per cent confidence limits for the mean values of $1 / \Delta \mathrm{C}_{\mathrm{H}_{2} \mathrm{O}}$.

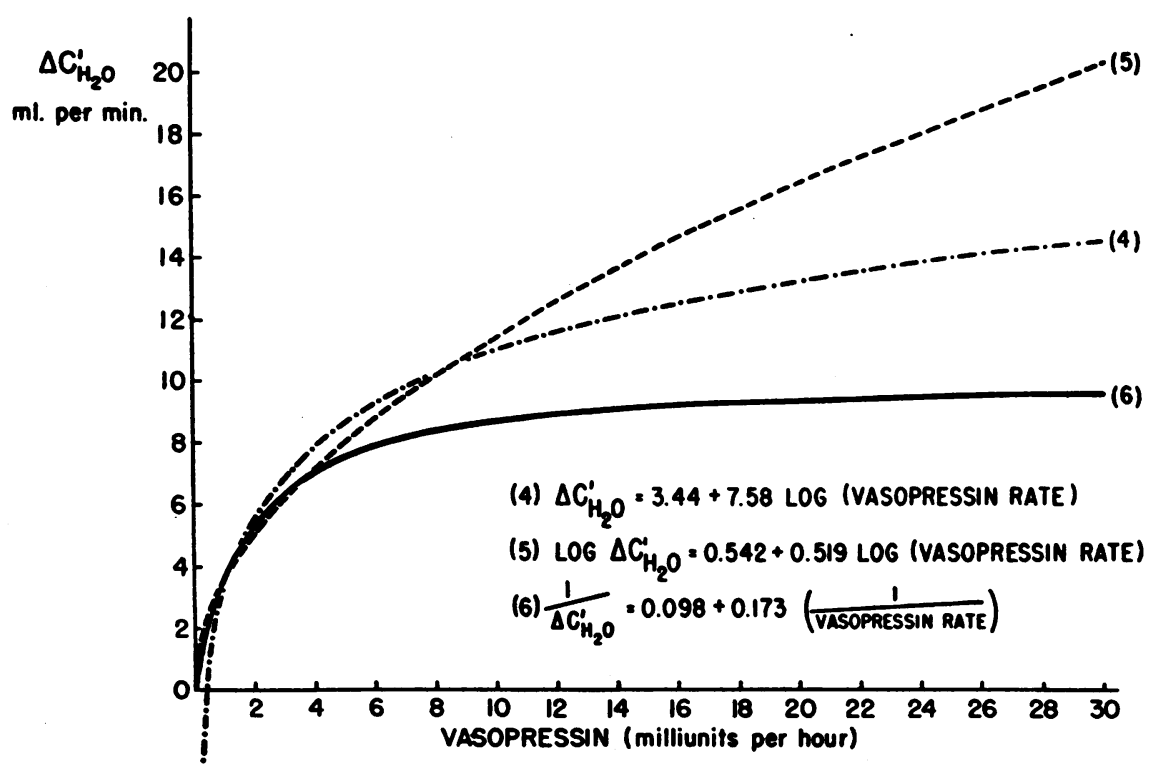

Fig. 3. Equations 4, 5, ANd 6 Extrapolated in Both Directions Frox the Range of the Actual Data 


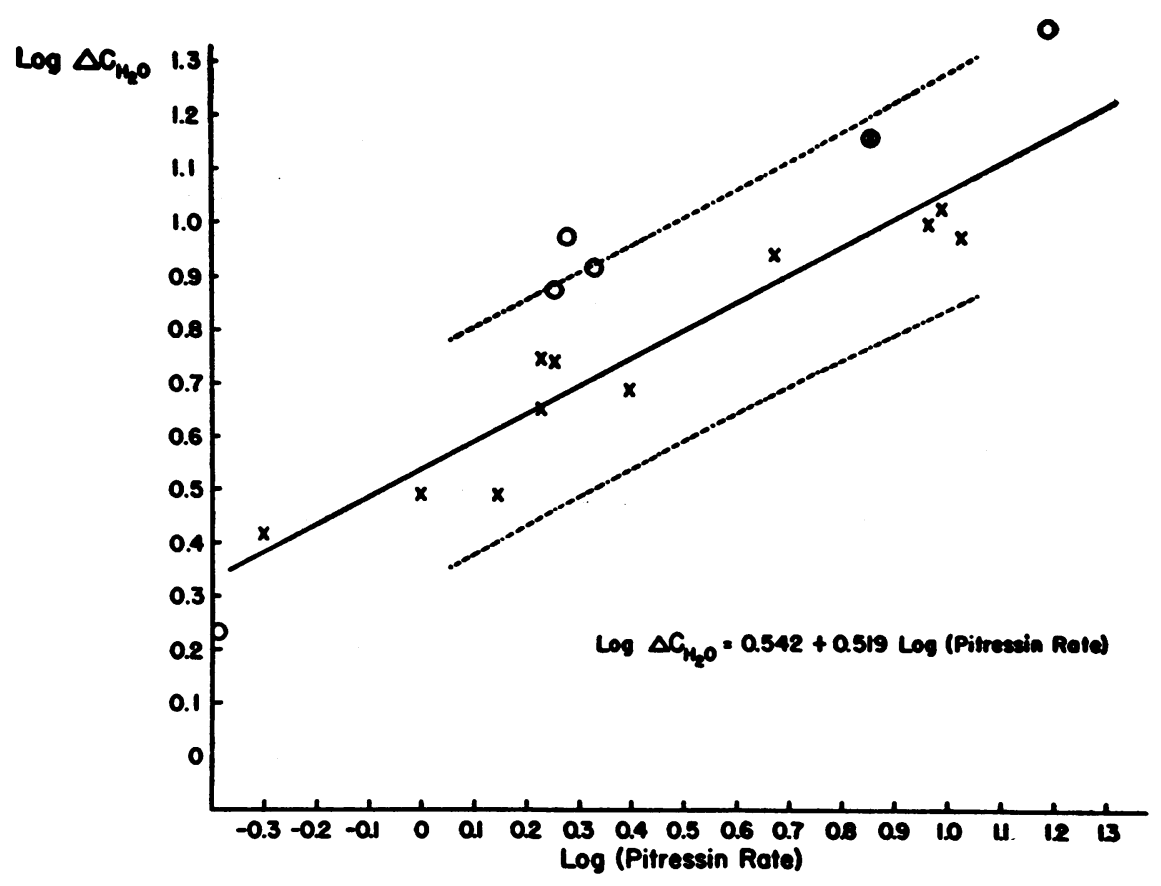

Fig. 4. Same as Figure 1 but Also Showing the Five Data From Studies in Which $\Delta \mathrm{C}_{\text {oem }}$ Was Greater Than 0.5 ML. Per Minute

Points designated with a circle represent the six studies associated with $\mathrm{C}^{\circ}{ }_{\text {oem }}>3.5 \mathrm{ml}$. per minute and/or $\Delta C_{\text {oum }}>0.5 \mathrm{ml}$. per minute. An augmented effect of vasopressin is apparent in five of the six.

during maximal water diuresis $\left(\mathrm{C}^{\circ}{ }_{\text {osm }}\right)$, and it appeared that with increasing values of $\mathrm{C}^{\circ} \mathrm{om}$ there was a slight increase in the $\Delta \mathrm{C}_{\mathrm{H}_{2} \mathrm{O}}^{\prime}$ for any particular rate of infusion of vasopressin. However, of the five experiments with the highest values of $\mathrm{C}^{\circ}$ oom $(>3.5 \mathrm{ml}$. per minute), four were associated with values of $\Delta \mathrm{C}_{\text {osm }}$ greater than 0.5 ml. per minute. Therefore, either $C_{\text {osm }}^{\circ}, \Delta C_{\text {osm }}$ or both (or some other related variable) appeared to have a significant effect on the renal response to vasopressin. This is illustrated in Figure 4.

One significant complication occurred in relation to these experiments. During a number of studies which are not included in the results reported, reactions occurred which were characteristic of those due to intravenous pyrogen (shaking chills or chilly feelings, temperature elevations from 100 to $103^{\circ} \mathrm{F}$. by mouth, and variable associated symptoms such as headache, nausea, vomiting, and muscular aches). These reactions were ultimately shown to be the result of the infusion of large volumes of commercially produced "pyrogen-free" parenteral fluid. This fluid was always found to be bacteriologically sterile and it is presumed that it contained minute amounts of pyrogenic material, which were sufficiently small to escape detection by the standard U.S.P. rabbit test $(5,6)$ and which would not cause reactions when infused in the volumes of solution commonly used for clinical purposes, but which would be capable of producing reactions if infused in large volumes at a rapid rate $(5,6)$ as in the studies recorded here (5 to 9 liters at 15 to $40 \mathrm{ml}$. per minute).

"Pyrogenic" reactions did occur during the 12 hours after completion of the study in four of the experiments included in this report (R.S. 8/22/ 54; R.W. $10 / 17 / 54$; E.D. $1 / 10 / 55$; and D.R. $2 / 23 / 55$ ). One subject (D.D. 8/26/54) became nauseated and vomited several times during the 6 hours after completion of the study but did not develop fever.

\section{DISCUSSION}

Vasopressin (Pitressin ${ }^{(8)}$ ) is assayed in terms of pressor activity, but since vasopressin and antidiuretic hormone are probably identical (7), the pressor and antidiuretic activities should 
bear a consistent quantitative relationship to each other. It is therefore reasonable to combine results obtained from two lots of Pitressin ${ }^{8}$, the pressor activity of each lot being known. Obviously, there is no particular significance in the absolute values of the rates of infusion since these necessarily have been assigned in terms of the biologically assayed "pressor" activity. Also, these calculated rates of infusion of vasopressin are only approximations, since the assay method is not accurate to better than \pm 7 per cent (8), and since the measurements involved in preparing the dilutions are subject to a small error.

Infusions of vasopressin at rates even considerably greater than those used in the current studies do not cause any large or consistent changes in the rate of glomerular filtration (clearance of inulin) (9) and cause slight if any alteration in total effective renal plasma flow (clearance of para-amino-hippurate) (9). Hence, it is unlikely that hemodynamic effects were involved in the current results, although such effects cannot be as clearly eliminated in the four experiments in which a pyrogenic reaction developed subsequent to (but not during) the study.

There are three fundamental assumptions implicit in the experimental design of these studies, the validity of which cannot be known: 1) That there was no significant secretion of endogenous $\mathrm{ADH}$ during "equilibrium" periods of what has been called "maximal water diuresis"; 2) that the changes in $\mathrm{C}_{\mathrm{H}_{2} \mathrm{O}}$ observed with the infusions of vasopressin were due entirely to the administered vasopressin; and 3) that when vasopressin is being infused at a constant rate, and when $\mathrm{C}_{\mathrm{H}_{2} \mathrm{O}}$ is constant, the amount of vasopressin acting on the appropriate site in the renal tubules is also constant. These assumptions are, at least, supported by the approximate constancy of $\mathrm{C}_{\mathrm{H}_{3} \mathrm{O}}$ during the "equilibrium" periods.

If the volume of distribution of vasopressin does not alter, and if the rate constant ${ }^{6}$ of biological decay of vasopressin is independent of concentration (as Ginsburg and Heller (10) found it to be in the rat), the concentration of vasopressin in the blood at equilibrium would be a

The rate constant is the proportion of the total quantity of $\mathrm{ADH}$ present in its volume of distribution which leaves that compartment by any route in unit time. linear function of the rate of infusion. Furthermore, if the blood flow to the affected portion of the renal tubules does not alter, the concentration of vasopressin in the critical region would be expected to be a linear function of the concentration in the blood and therefore of the rate of infusion. Within the context of these and the aforementioned assumptions, one of the three types of derived regression equations may be a plausible expression of the physiologic process by which ADH effects the abstraction of water from the renal tubular urine. Unfortunately, establishment of such mathematical relationships cannot of itself prove the existence of any particular mechanism, and in the discussion which follows several general concepts with respect to mechanism will be examined without intending to suggest that those mentioned in any sense represent an inclusive list.

Equations 1 and 4 express an exponential type of relationship which can be presented in the form $10^{\Delta C^{\prime} H_{2} \mathrm{O}}=A$ (Vasopressin rate) ${ }^{B}$. This, of course, is a commonly used type of dose-response equation, and if appropriate for the current data, it does not provide any particular insight with respect to possible mechanisms. Since this form of mathematical expression predicts unlimited values of $\Delta C^{\prime}{ }_{H_{3} \mathrm{O}}$ at ever increasing rates of infusion of vasopressin, it obviously cannot be applicable much above the range actually studied. Furthermore, this type of equation predicts theoretically negative values for $\Delta C_{H_{2} O}^{\prime}$ (which approach minus infinity as a limit) as the rate of infusion of vasopressin approaches zero as a limit, which is almost certainly not in conformity with reality. ${ }^{7}$

Equations 2 and 5 express an exponential relationship of the type $\Delta \mathrm{C}^{\prime}{ }_{\mathrm{H}, \mathrm{O}}=\mathrm{A}$ (vasopressin rate $)^{B}$ and, since the exponent is approximately 0.5 , imply that the reabsorption of water is proportional to approximately the square root of the rate of infusion of vasopressin. This is reminiscent of Schütz's Law $(11,12)$ which states

${ }^{7}$ It is implicit in the experimental design of these studies that a rate of infusion of vasopressin of zero evokes a zero response $\left(\Delta C^{\prime}{ }_{H_{2} O}=0\right)$. Although the semi-logarithmic form of equation appears valid within the range of the actual experimental data, such an expression cannot define accurately the theoretical dose-response relationship in the range where the dose is approaching zero and when $\Delta C^{\prime}{ }_{H_{2} O}$ also would be expected to be approaching zero. 
that the rate of certain enzymatic reactions is a function of the square root of the enzyme concentration, ${ }^{8}$ thereby suggesting the possibility that ADH may act as an enzyme or apo-enzyme for a chemical reaction effecting the reabsorption of water. Since this type of equation also predicts unlimited values of $\Delta C^{\prime}{ }_{\mathrm{H}_{2} \mathrm{O}}$ at ever increasing doses of vasopressin, it (like equations 1 and 4) cannot be applicable much above the range studied.

Relationships of the type represented by equations 3 and 6 can be derived from several hypothetical modes of action of ADH. One such derivation ${ }^{9}$ has been suggested by Dr. Hans Ussing (13) who proposes that each molecule of $\mathrm{ADH}$ is potentially able to combine reversibly in some way with a "site" in the renal tubular cell or on its membrane, thereby creating a "pore" through which water can flow at a constant rate. The actual mechanism causing the flow of water is immaterial; the term "pore" merely indicates the rate-limiting element of the process. It is further assumed that the total number of potential "pores" is constant, that all potential "pore" sites have the same affinity for $\mathrm{ADH}$, and that the rate of movement of water is proportional to the number of "pores." From these assumptions it is possible to derive the following equation : ${ }^{9}$

$$
\frac{1}{\Delta \mathrm{W}}=\frac{1}{\mathrm{M}_{0} \mathrm{a}}+\frac{\mathrm{k}}{\mathrm{M}_{0} \mathrm{a}}\left(\frac{1}{\mathrm{H}}\right)
$$

where

$$
\begin{aligned}
\Delta \mathrm{W}= & \text { rate of reabsorption of water resulting } \\
& \text { from ADH. } \\
\mathrm{H}= & \text { number of available ADH molecules. } \\
\mathrm{k}= & \text { the dissociation constant for the com- } \\
& \text { bination of vasopressin with the so- } \\
& \text { called "pore" sites. } \\
\mathrm{M}_{0}= & \text { total number of potential "pores" (con- } \\
& \text { stant). } \\
\mathrm{a}= & \text { constant. }
\end{aligned}
$$

Assuming that $\Delta \mathrm{W}$ represents $\Delta \mathrm{C}_{\mathrm{H}_{2} \mathrm{O}}^{\prime}$ and that $\mathrm{H}$ is proportional to the rate of infusion of vasopressin, this is comparable to equations 3 and 6.

The conceptual approach suggested by Ussing

\footnotetext{
8 There appears to be considerable uncertainty with respect to the validity of this "law" for any enzymatic reaction.

- See Appendix A.
}

would apply to any mechanism which satisfied the same general assumptions; i.e., the reversible combination of $\mathrm{ADH}$ with some "receptor" in or on the renal tubular cells, a limited number of receptors, and a linear relationship between the number of $\mathrm{ADH}$-receptor combinations and the rate of reabsorption of water. Furthermore, it is apparent that these assumptions and the reciprocal type of equation derived from them are essentially analogous to the Lineweaver-Burk expression of the Michaelis-Menten theory of enzyme kinetics. The possible usefulness of this approach to the physiologic interpretation of hormone dose-response relationships has also been recently suggested by Stetten (14).

Another concept from which it is possible to derive a relationship of the type expressed in equations 3 and 6 is that the effect of $\mathrm{ADH}$ is, in some unknown way, to raise the osmotically effective concentration in the renal tubular cells (thus promoting the osmotic reabsorption of water), and that each molecule of ADH results in a constant increment of that effective concentration up to a maximum. ${ }^{10}$

It will be noted that equations of the type just discussed, unlike equations $1,2,4$, and 5 , predict that at increasing rates of infusion of vasopressin, $\Delta \mathrm{C}_{\mathrm{H}_{2} \mathrm{O}}$ will approach a maximum $\left(\mathrm{M}_{\mathrm{Oa}}\right)$ asymptotically. It might therefore be implied that the process effected by $\mathrm{ADH}$ is limited by a maximum rate, an implication which is consistent with the current hypothesis $(3,15,16)$ in which the "facultative" reabsorption of water by the renal tubules is considered to be a dual process involving: 1) $\mathrm{T}^{\mathrm{d}} \mathrm{H}_{2} \mathrm{O}$, the rate of reabsorption of water in the distal tubule, under the influence of ADH but limited by the rate of reabsorption of solutes since its maximum is that which will produce a concentration isosmotic with the glomerular filtrate; and 2) $\mathrm{T}^{\circ}{ }_{\mathrm{H}_{2} \mathrm{O}}$, the rate of reabsorption of solute-free water at some site distal to that of $\mathrm{T}^{\mathrm{d}} \mathrm{H}_{2} \mathrm{O}$, presumably not influenced by $\mathrm{ADH}$, approximately constant and maximal $\left(\mathrm{Tm}^{\circ}{ }_{\mathrm{H}_{2} \mathrm{O}}\right)$ at relatively high rates of solute excretion or whenever the final urine formed is hypotonic to plasma. If such a dual mechanism were in fact operative, the measured values of $\Delta \mathrm{C}_{\mathrm{H}_{2} \mathrm{O}}$ in the current studies could be represented as follows: $\Delta \mathrm{C}_{\mathrm{H}_{2} \mathrm{O}}=\Delta \mathrm{T}^{\mathrm{d}}{ }_{\mathrm{H}_{2} \mathrm{O}}+\Delta \mathrm{T}^{\mathrm{o}}{ }_{\mathrm{H}_{2} \mathrm{O}}$. Since the

${ }^{10}$ See Appendix B. 
final urine was almost always hypotonic to the plasma, $\mathrm{T}^{\circ}{ }_{\mathrm{H}, \mathrm{O}}$ should equal $\mathrm{Tm}^{\circ}{ }_{\mathrm{H}, \mathrm{O}}$, and as the latter is presumably constant, it could be assumed that, within the range studied, $\Delta \mathrm{C}_{\mathrm{H}_{2} \mathrm{O}}=\Delta \mathrm{T}^{\mathrm{d}}{ }_{\mathrm{H}_{2} \mathrm{O}}$. For this reason, it might be appropriate to consider the derived regression equation as a description of the relationship between the rate of infusion of vasopressin and $\Delta \mathrm{T}^{\mathrm{d}}{ }_{\mathrm{H}, 0}$. In this regard, it is interesting that the maximum values of the dependent variable predicted by equations 3 and 6 (14.1 and $10.2 \mathrm{ml}$. per minute, ${ }^{11}$ respectively) are of the same order of magnitude as that anticipated for $\Delta T^{d}{ }_{n, 0}$ from calculations based on the dual mechanism hypothesis ${ }^{12}$ (15.8 ml. per minute $\left.{ }^{11}\right)$. Thus, although there is no particular basis for assuming that the relationship between $A D H$ and $\Delta T^{d}{ }_{H_{2}} O$ must be described by a single continuous regression equation, it appears that the reciprocal form (equations 3 and 6 ) is the only one of the three simple types discussed here which might reasonably be applicable much above the range of the current data.

The renal response to vasopressin or to an extract of the posterior pituitary gland has been examined in many animal studies (1, 2, 17-22). Most of these cannot be readily compared with the present experiments because of differences in technique and because the dose-response relationship often is uncertain. Nonetheless, at least two studies do allow the derivation of a quantitative relationship between several sub-maximal doses of injected material and the renal response. Ginsburg and Heller (22) examined the antidiuretic action of individual doses of vasopressin injected intravenously into unanesthetized rats. Table IV is based on Figure 1 of their report. Change in urine flow has been calculated from the flows shown before, and for varying periods after, injection of vasopressin. Maximum change in flow, $\Delta$ flow $_{\max }$, is based on the lowest urine flow during any single period following the injection. $\Delta$ flow $_{20}$ and $\Delta$ flow $_{30}$ are based on aver-

${ }^{11}$ All figures corrected to body surface area of 1.73 square meters.

${ }^{12}$ From Zak, Brun, and Smith (16) $\mathrm{Tm}^{\circ}{ }_{\mathrm{H}_{2} \mathrm{O}}$ in waterloaded adults $=4.1 \mathrm{ml}$. per minute. The average maximum $\mathrm{C}_{\mathrm{H}_{2} \mathrm{O}}$ in the current studies $=11.7 \mathrm{ml}$. per minute. The theoretically maximum $\Delta \mathrm{T}^{\mathrm{d}} \mathrm{H}_{2} \mathrm{O}$ is the sum of $\mathrm{Tm}^{\circ} \mathrm{H}_{2} \mathrm{O}$ and the maximum $\mathrm{C}_{\mathrm{B}_{2} \mathrm{O}}=4.1+11.7=15.8 \mathrm{ml}$. per minute.
TABLE IV

Renal response of rats to vasopressin (from Ginsburg and Heller [22] Figure 1)

\begin{tabular}{cccc}
\hline \hline $\begin{array}{c}\text { Dose of } \\
\text { vasopressin }\end{array}$ & $\Delta$ flowmax & $\Delta$ flowrot & $\Delta$ flowsof \\
\hline (milliunits) & ml./10 min. & ml. $/ 10$ min. & ml. $/ 10$ min. \\
0.1 & 0.6 & 0.4 & 0.4 \\
0.2 & 1.0 & 0.85 & 0.6 \\
0.4 & 1.4 & 1.3 & 0.9 \\
0.6 & 1.8 & 1.65 & 1.3 \\
0.8 & 1.8 & 1.75 & 1.3
\end{tabular}

* $\Delta$ flow $_{\max }=$ Difference between pre-injection flow and minimum flow during any ten-minute period following injection.

$\dagger \Delta$ flow $_{20}=$ Difference between pre-injection flow and the average flow during first twenty minutes following injection.

$\ddagger \Delta$ flow $_{30}=$ Same as $\Delta$ flow $_{20}$ except post-injection flow averaged for thirty minutes.

ages obtained by including the urine flows for 20 and 30 minutes, respectively, after the injection of vasopressin. As the authors state, a plot of the logarithm of the dose against the response is well described by a straight regression line. As shown in Figure 5, the data (using $\Delta$ flow $_{30}$ ) are also well defined by either a log-log or reciprocal relationship. The regression coefficients are quite similar to those reported here: 0.601 for the $\log -\log$ (compared to 0.564 and 0.519 in equations 2 and 5); 0.215 for the reciprocal relationship (compared to 0.196 and 0.173 in equations 3 and 6). Jeffers, Livezey, and Austin (19) studied the dose-response relationship of vasopressin injected intravenously (as individual doses) into water-loaded rats receiving alcohol, the response being defined as the difference between urine flow for 20 minutes after and 20 minutes before injection. Again, the results are well expressed by either a semi-logarithmic or a log-log type of regression equation, and somewhat less well by one involving reciprocals. The studies of Lauson (2) on a normal man and those of Shannon (1) on dogs with diabetes insipidus have been mentioned in the introduction. Both of these investigators utilized a technique which was similar to the one employed in the current experiment but the range studied generally was higher than in the current studies.

\section{SUMMARY AND CONCLUSIONS}

An attempt has been made to define the quantitative relationship between antidiuretic hor- 

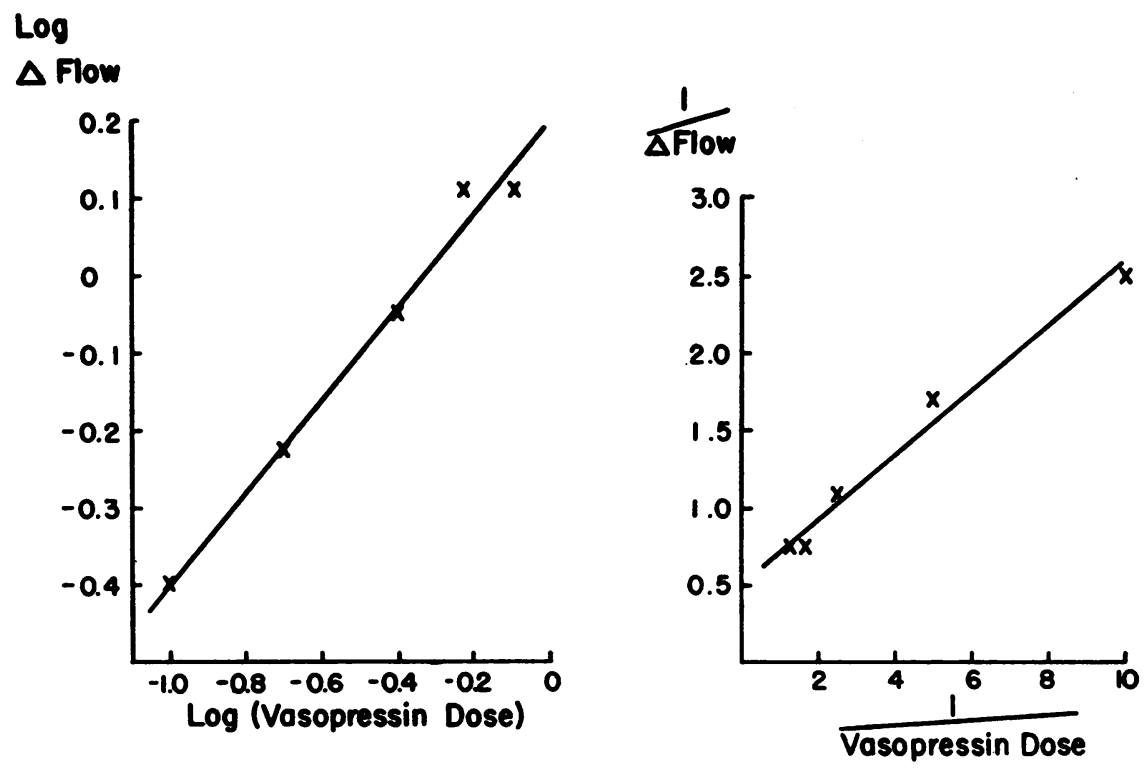

Fig. 5. The Relationship Between $\Delta$ Flow (Ml. per 10 Minutes) and the Dose of Vasopressin (Milliunits) in Rats, Based on Data Obtained by Ginsburg and Heller (22), See TeXT

mone (vasopressin) and the renal tubular reabsorption of water in continuously water-loaded young adult men. The vasopressin was infused at various constant rates, almost all of which were less than that required to produce an hypertonic urine. The difference between the clearance of free water during maximal water diuresis and the clearance of free water during an infusion of vasopressin $\left(\Delta \mathrm{C}_{\mathrm{H}_{2} \mathrm{O}}\right)$ was considered to represent the rate of reabsorption of water resulting from the particular rate of infusion of vasopressin. The results indicate that there is a graded curvilinear relationship between the rate of infusion of vasopressin and the renal tubular reabsorption of water. The relationship can be expressed by at least three simple regression equations, the possible physiologic significance of which has been discussed. It is suggested that an equation of the form $1 / \Delta \mathrm{C}_{\mathrm{H}_{2} \mathrm{O}}=\mathrm{A}+\mathrm{B}$ (1/ vasopressin rate) may be the most physiologically meaningful expression of the data.

\section{ACKNOWLEDGMENTS}

The authors wish to thank Mr. Edmund Gehan of the Department of Biostatistics, Dr. Harry Smith, formerly of the same department, and Dr. Robert Mann of the Department of Mathematics, all of the University of North Carolina and all of whom gave considerable assistance with statistical and mathematical considerations.

\section{REFERENCES}

1. Shannon, J. A., The control of the renal excretion of water. II. The rate of liberation of the posterior pituitary antidiuretic hormone in the dog. J. Exper. Med., 1942, 76, 387.

2. Lauson, H. D., The problem of estimating the rate of secretion of antidiuretic hormone in man. Am. J. Med., 1951, 11, 135.

3. Wesson, L. G., Jr., and Anslow, W. P., Jr., Effect of osmotic and mercurial diuresis on simultaneous water diuresis. Am. J. Physiol., 1952, 170, 255.

4. Williams, T. F., Hollander, W., Jr., Strauss, M. B., Rossmeisl, E. C., and McLean, R., Mechanism of increased renal sodium excretion following mannitol infusion in man. J. Clin. Invest., 1955, 34, 595.

5. Dare, J. G., and Mogey, G. A., Rabbit responses to human threshold doses of a bacterial pyrogen. J. Pharm. \& Pharmacol., 1954, 6, 325.

6. Co Tui, F. W., Practical aspects of the pyrogen problems. Proc. Am. Pharm. Mfrs. Assoc., Mid-year meeting, 1943, 40.

7. Du Vigneaud, V., Hormones of the posterior pituitary gland: Oxytocin and vasopressin. The Harvey Lectures, 1954-55, New York, Academic Press, 1956, p. 1.

8. Bratton, A. C., Jr., Personal communication.

9. Maxwell, M. H., and Breed, E. S., The effect of the intravenous administration of Pitressin on renal function in man. J. Pharmacol. \& Exper. Therap., 1951, 103, 190.

10. Ginsburg, M., and Heller, H., The clearance of injected vasopressin from the circulation and its fate in the body. J. Endocrinol., 1953, 9, 283. 
11. Sumner, J. B., and Myrbäck, K., Ed., The Enzymes; Chemistry and Mechanism of Action. New York, Academic Press, 1950, p. 64.

12. Riggs, D. S., Quantitative aspects of iodine metabolism in man. Pharmacol. Rev., 1952, 4, 284.

13. Ussing, H., Personal communication.

14. Stetten, DeW., Jr., Relationship of hormone dosage to physiological response. Science, 1956, 124, 365.

15. Smith, H. W., The Kidney: Structure and Function in Health and Disease. New York, Oxford University Press, 1951.

16. Zak, G. A., Brun, C., and Smith, H. W., The mechanism of formation of osmotically concentrated urine during the antidiuretic state. J. Clin. Invest., 1954, 33, 1064.

17. Samaan, A., The effect of pituitary (posterior lobe) extract upon the urinary flow in non-anaesthetized dogs. J. Physiol., 1935, 85, 37.

18. Ingram, W. R., Ladd, L., and Benbow, J. T., The excretion of antidiuretic substance and its relation to the hypothalamico-hypophyseal system in cats. Am. J. Physiol., 1939, 127, 544.

19. Jeffers, W. A., Livezey, M. M., and Austin, J. H., A method for demonstrating an antidiuretic action of minute amounts of Pitressin: Statistical analysis of results. Proc. Soc. Exper. Biol. \& Med., 1942, 50, 184.

20. Ames, R. G., and Van Dyke, H. B., Antidiuretic hormone in the serum or plasma of rats. Endocrinology, 1952, 50, 350.

21. Stein, M., Jinks, R., and Mirsky, I. A., The bioassay of Pitressin and antidiuretic substances in blood and urine. Endocrinology, 1952, 51, 492.

22. Ginsburg, M., and Heller, H., The antidiuretic assay of vasopressin by intravenous injection into unanaesthetized rats. J. Endocrinol., 1953, 9, 267.

\section{APPENDIX A}

Derivation of equation based on "pore" theory of Ussing (13). Let:

$\mathrm{H}=$ Available ADH molecules.

$\mathbf{M}_{0}=$ Total number of "sites" potentially able to combine with ADH to form "pores."

M = Number of "sites" not combined with ADH.

$\mathrm{HM}=$ Number of "pores" (H and $\mathrm{M}$ combinations).

$\Delta W=$ Rate of reabsorption of water resulting from ADH.

$\mathrm{a}=$ Constant .
Assume:

1) That combination of $\mathrm{H}$ and $\mathrm{M}$ is reversible and obeys the law of mass action;

2) That $\Delta W$ is proportional to the number of "pores."

Then:

$$
\begin{aligned}
& \mathbf{M}_{0}=\mathbf{M}+\mathrm{HM} \\
& \mathbf{H}+\mathbf{M} \rightleftharpoons \mathrm{HM} \\
& \frac{\mathbf{H} \times \mathbf{M}}{\mathbf{H M}}=\mathbf{k} \\
& \Delta \mathrm{W}=\mathbf{a}(\mathrm{HM})
\end{aligned}
$$

Substituting from (I) and (IV) in (III):

$$
\frac{H\left(M_{0}-\frac{\Delta W}{a}\right)}{\frac{\Delta W}{a}}=k
$$

Rearranging (V):

$$
\frac{1}{\Delta \mathrm{W}}=\frac{1}{\mathrm{M}_{0} \mathrm{a}}+\frac{\mathrm{k}}{\mathrm{M}_{0 \mathrm{a}}}\left(\frac{1}{\mathrm{H}}\right)
$$

Let :

\section{APPENDIX B}

$\mathrm{H}=$ Available ADH molecules.

$\Delta W=$ Rate of reabsorption of water resulting from ADH.

$\Delta$ osm $=$ Increased osmolality of renal tubular cells resulting from ADH.

$a=$ Effective osmolality of renal tubular cells in absence of $\mathrm{ADH}=$ constant.

b = Rate at which renal tubular urine is delivered to site where ADH is effective = constant.

$\mathbf{k}=$ Constant.

Assume :

1) That renal tubular urine will always become isosmotic with the renal tubular cells $=a+\Delta o s m$;

2) That the rate of reabsorption of solutes is constant ;

3) That $\Delta$ osm is proportional to $\mathrm{H}$.

Then:

$$
\Delta \mathrm{W}=\mathrm{b}-\frac{\mathrm{ab}}{\mathrm{a}+\Delta \mathrm{osm}}
$$

Rearranging (I) :

$$
\frac{1}{\Delta \mathrm{W}}=\frac{1}{\mathrm{~b}}+\frac{\mathrm{a}}{\mathrm{b}}\left(\frac{1}{\Delta \mathrm{osm}}\right)
$$

From assumption (3):

$$
\frac{1}{\Delta W}=\frac{1}{b}+\frac{a k}{b}\left(\frac{1}{H}\right)
$$

